\title{
International migrations and urbanisation: \\ 1960-2010
}

\author{
Vicente Royuela* and \\ David Castells-Quintana \\ AQR-IREA Research Group, \\ Universitat de Barcelona, \\ Av. Diagonal 690, 08034 Barcelona, Spain \\ E-mail:vroyuela@ub.edu \\ E-mail: dcastells@ub.edu \\ *Corresponding author
}

\begin{abstract}
International migrations and urbanisation rates have seen a large increase in the last decades. Here we analyse the relationships between migrations and urbanisation by using a panel of $\mathrm{ca} 200$ countries over the period 1960-2010. We describe the main global stylised facts on urbanisation and international migrations focusing on differences in these across world regions. We found that while there was a positive association between immigration and urbanisation, particularly in small and medium-sized cities, the association between emigration and urbanisation in developing countries was inverse. Both associations have become stronger over the few past decades, and our results highlight that international migration is an increasingly relevant and complementary dimension of the traditional rural-urban reallocation of workers which takes place during economic development.
\end{abstract}

\section{Keywords}

Urbanisation; international migration; urban concentration; development

\section{JEL Classification}

R00, R23, F22

\section{Acknowledgements}

The authors gratefully acknowledge financial support from the European Commission under the 7th Framework Programme project SEARCH, FP7-SSH-2010-2.2-1-266834 and also acknowledge the financial support of CICYT ECO2010-16006. 


\section{Introduction}

In 1960 one third of the world's population lived in cities. In 2012 this figure reached 52.6\%. At this speed of growth, which has been particularly faster in the last two decades, in 2050 around two thirds of the world population will be living in cities. The process of urbanisation is related to the process of economic development, with people migrating from lagged rural areas to developing urban ones, which exhibit higher productivity and offer higher wages. However, migration flows currently occur not just within countries but also increasingly between them, with people also looking for opportunities, derived for instance, by international differentials in productivity levels and real wages. Migrations have important consequences on both sending and receiving countries. In fact, despite the fact that the largest flows have been within countries, the number of persons living outside their country of birth has surpassed 200 million people (almost three per cent of the world's population in 2010). While in OECD countries, the population of foreign origin now represents about $12 \%$ of the total population (i.e. are net 'importers' of people), in other parts of the world, such as Central Asia, Central America and the Caribbean, more than 10\% of the population has migrated out of their country. Acknowledging these two global trends, the aim of this paper has been to inspect whether international migration can be considered as a relevant factor in the evolution of the internal economic geography of countries.

In this work, we analyse the increasing role of cities all over the world, and the importance of international migrations in the process of urbanisation, using a wide database of 197 countries over the period 1960-2010. We focused on differences across world geographical regions in levels of economic development and rates of urbanisation. Urbanisation was examined both quantitatively (i.e. rates) and qualitatively, i.e. by considering urbanisation in small and-medium sized cities and the process of urban concentration in larger agglomerations.

The paper is structured in five sections. After this introduction we present a brief literature review on the determinants of urbanisation, focusing on a potential role for international migrations, which prepares the ground for inspecting the current trends in these two processes through correlation analyses (section 3). In section 4 we use a parsimonious econometric model for urbanisation. Finally, we discuss the main findings and conclusions.

\section{Determinants of urbanisation: A brief literature review}

The central role of urbanisation in the process of economic development is extensively present in the literature (Lewis, 1954; Ranis and Fei, 1961; Harris and Todaro, 1970; Todaro, 1976, to mention a few seminal papers). In most of these models, migration occurs between lagged rural areas and developed urban areas, driven by the higher wages in the latter due to the higher productivity of the urban sector. As migration proceeds, the economy experiences a structural change moving from 
agricultural-based activities to industrial activities, which are the base for economic development (Gollin et al. 2002). However, urbanisation, and urban concentration in large agglomerations, is also maintained by the higher productivity driven by agglomeration economies (even at the risk of urban underemployment, as expressed in Rauch, 1993), with labour mobility being the human side of the agglomeration story. Urbanisation can be seen as a manifestation of development processes, and "migration is a contributor of development, a corrector of regional imbalances, and a conqueror of the tyranny of space," in the words of Firebaugh (1979, p.199).

More recent literature has focused on the process of urbanisation itself, considering not just urbanisation but also urban concentration. In this line, there are papers providing theoretical modelling (Henderson and Wang, 2005), as well as empirical evidence on the association between the sector shift out of agriculture and urbanisation (Brueckner, 1990; Davis and Henderson, 2003; Castells-Quintana and Royuela, 2011 and Franch Auladell et al., 2013). Kasarda and Crenshaw (1991) define three proximate determinants of urban growth: the natural increase of urban population; boundary redefinition through annexation of surrounding areas; and migration, both intra-national (rural-urban and urban-urban) and international. However, as the authors highlight, scholars have focused mainly on rural-urban migration rather than on international migration. The relevant role of other factors driving urbanisation has also been highlighted, including institutions (Henderson and Wang, 2007), adverse rural conditions (Firebaugh, 1979) and climate change especially in Sub-Saharan Africa (Barrios et al., 2006). Finally, regarding urban concentration, other factors have been found to be relevant. Ades and Glaeser (1995) report as significant determinants, high tariffs, high costs of internal trade and low levels of international trade, as well as political factors, while Davis and Henderson (2003) analyse the role of specific policies like development of interregional infrastructure and fiscal decentralization.

International migrations are an increasingly important factor accompanying traditional internal migrations. However, they are often overlooked in the analyses of urbanisation processes. International migrations can be understood as an equaliser of the marginal productivity of labour not just between rural and urban spaces, but also between countries. Additionally, in the same way as rural-urban migrations within countries, associations are also expected between international migrations and features of the process of economic development and thus also with changes in the economic geography of countries. Of course, we expect migration processes to affect the economic geography of both sending as well as receiving countries.

Overall, as the World Development Report (World Bank, 2009) stresses, "an important insight of the agglomeration literature - that human capital earns higher returns where it is plentiful - has been ignored by the literature of labour migration" (WDR, 2009, p. 158). Novel understandings come 
from considering together agglomeration economies and labour migration, which takes place both at the national and at the international level.

\section{Data, trends and analysis of the relationship between urbanisation and international migration}

\subsection{Data and trends}

In this section we describe the main trends in urbanisation and international migrations all over the world by exploring variables on population and urbanisation gleaned from the World Bank World Development Indicators at the country level. ${ }^{1}$ Regarding migration, our data sources were the World Bank Bilateral Migration Database 1960-2000 and the World Bank Bilateral Migration Matrix 2010. Data are from 197 countries for the years 1960, 1970, 1980, 1990, 2000 and 2010. ${ }^{2}$ These datasets provide information on stocks rather than on flows. However, we selected these data because immigration stocks data are based on national censuses, and therefore are probably of higher quality than data reporting annual immigrant flows. Censuses deal with unambiguous, net permanent moves. In our paper we have followed Ortega and Peri (2009), who argue that theoretical models of migration can be interpreted as determining the relationships for stocks of migrants, or the analogous flows. As they do, we will consider the stock of migrants as a proxy of long-term migration flows.

Table 1 presents the main urbanisation trends by continent and world subregion. ${ }^{3}$ The urban world population has increased from 33\% in 1960 to 51\% in 2010. In Africa, America and Asia urbanisation has increased by at least 20 points, in Europe by 16, and in Oceania by 4 (already having a high rate in 1960). In 2010, 15 subregions had more than half of their population living in cities, while in the remaining eight regions the figure was still below 50\%. Urban concentration (defined as population living in cities of more than one million inhabitants) has globally risen by $6 \%$ in the last 50 years, being especially important in America, Oceania, and in several other subregions, such as Southern Africa and Western Asia. However, the global urbanisation trend has a stronger pace in small and medium-sized cities (i.e. less than one million inhabitants). These cities have grown two times faster than larger cities over the past 50 years (smaller cities: 20\% in 1960 to 32\% in 2010 vs. larger cities: $13 \%$ in 1960 to 19\% in 2010). In two regions, Central Asia and Northern Europe, large cities actually lost weight, while small and medium-sized cities were responsible for the entire increase in urbanisation rates. Moreover, in Europe we can see that more

\footnotetext{
${ }^{1}$ Urban population refers to people living in urban areas as defined by National Statistical Offices. It has been estimated from World Bank population estimates and urban ratios from the United Nations World Urbanisation Prospects.

2 The list of countries is reported in Annex 1. These databases can be respectively accessed at http://data.worldbank.org/data-catalog/global-bilateral-migration-database and http://go.worldbank.org/JITC7NYTT0. We used the version collected and revised by Ramos (2013). Over one thousand census and population register records have been combined to construct decennial matrices.

${ }^{3}$ Annex 1 classifies the countries by continent and geographical region.
} 
than $80 \%$ of the increase in urbanisation rates was due to the growth of small and medium-sized cities, rather than due to population increases in the large cities. Finally, the growth in urbanisation has been faster in recent decades, and this is true for both small/medium-sized $(<1$ million inhabitants) and large cities.

Table 2 shows the main demographics trends, also by continent and world subregion. Population growth has slowed down over the last 40 years, although several world subregions still had annual growth rates in excess of $2 \%$ in 2010, mainly African regions, Western Asia and Melanesia. Eastern Europe is the only subregion where population has decreased in the past two decades. Regarding migration, although the number of migrants has increased over time, its share of total world population was 3\% in 1960 and slightly decreased in 1990 (2.6\%), followed by resurgence since then $(2.8 \%$ in 2010$)$.

Immigration has been particularly pronounced in rich regions, like North America, most of Europe and Australia and New Zealand, compared to other regions. In North America, Western Europe, Australia and New Zealand, immigration largely exceeds emigration. On the other hand, emigration is more important in the Caribbean, Central America, Central Asia, Eastern Europe, Micronesia and Polynesia, with insular regions (such as the Caribbean and the Polynesia) showing the most dramatic increases in emigration rates from 1960 to 2010 and where emigration currently largely exceeds immigration. Interestingly, African regions, Western Asia and Melanesia, with the highest population growth, do not show the highest rates of emigration.

Overall, although urban population has increased in both small and medium-sized cities and in larger cities, among all urban people in the world the proportion of population living in large cities has decreased slightly over the last 50 years (39\% in 1960 to 37\% in 2010). We found important differences in this across world regions; while in certain regions urban concentration in large cities is substantial (e.g. North and South America, Southern Africa, Australia and New Zealand), in other regions it is the small and medium-sized cities that drives overall urban growth (similarly to European regions and Central Asia). Regarding international migrations, developed regions, which are usually the more urbanised ones, have the highest levels of net immigration (defined as the difference between immigration and emigration), while poorer regions show higher levels of net emigration. 
Table 1. World urbanisation trends per world regions. 1960-2010.

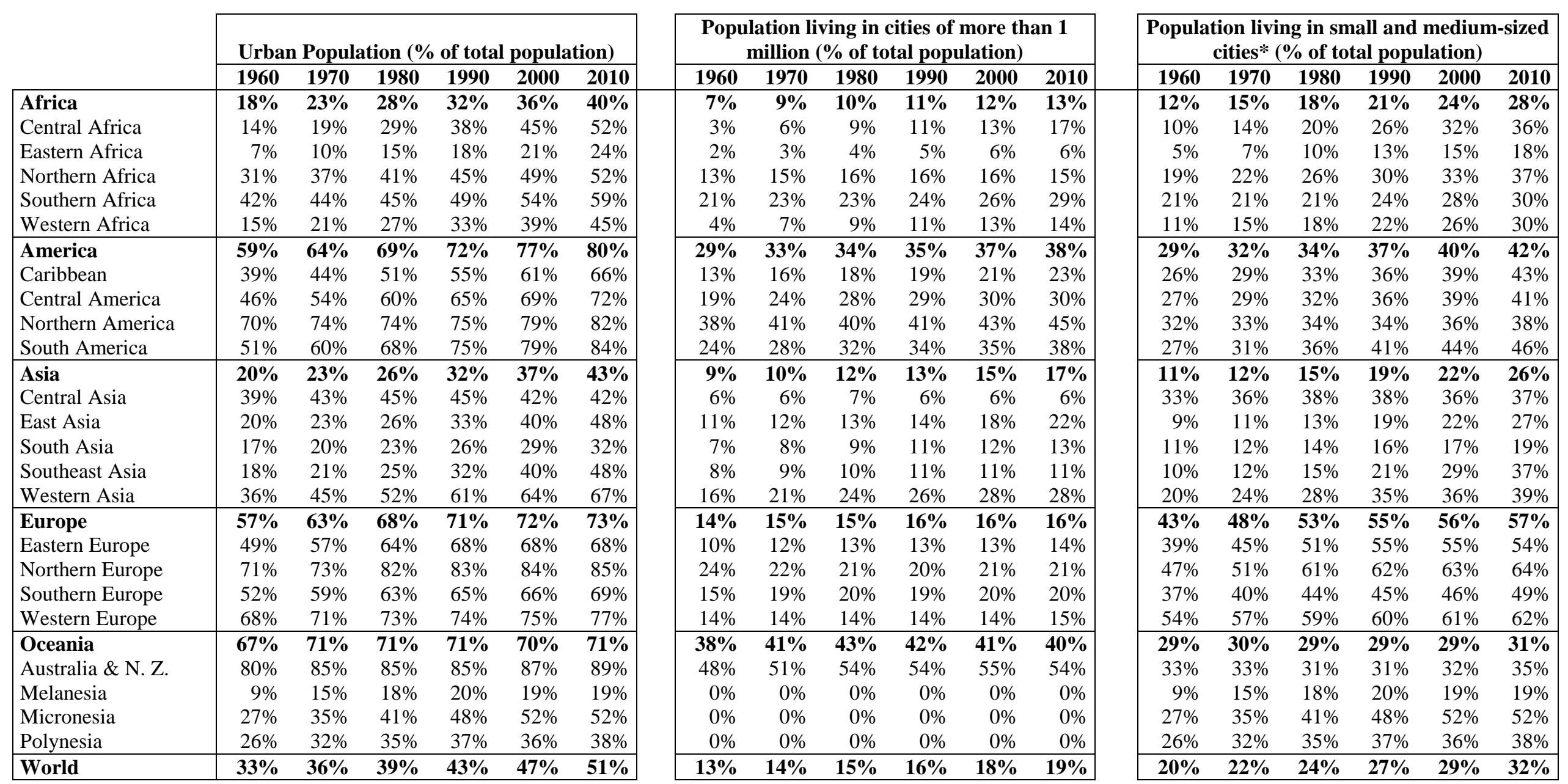

$*<1$ million inhabitants 
Table 2. World Demographic trends per world regions. 1960-2010.

\begin{tabular}{|c|c|c|c|c|c|c|c|c|c|c|c|c|c|c|c|c|c|}
\hline & \multicolumn{5}{|c|}{ Population Growth - annual rates } & \multicolumn{6}{|c|}{ Emigrants (as \% of local population) } & \multicolumn{6}{|c|}{ Immigrants (as \% of local population) } \\
\hline & $\begin{array}{l}1960- \\
1970\end{array}$ & $\begin{array}{c}1970- \\
1980 \\
\end{array}$ & $\begin{array}{c}1980- \\
1990 \\
\end{array}$ & $\begin{array}{l}1990- \\
2000 \\
\end{array}$ & $\begin{array}{l}2000- \\
2010\end{array}$ & 1960 & 1970 & 1980 & 1990 & 2000 & 2010 & 1960 & 1970 & 1980 & 1990 & 2000 & 2010 \\
\hline Africa & $2.5 \%$ & $2.7 \%$ & $2.8 \%$ & $2.5 \%$ & $2.3 \%$ & $2.9 \%$ & $2.9 \%$ & $2.9 \%$ & $2.6 \%$ & $2.5 \%$ & $2.9 \%$ & $2.9 \%$ & $2.2 \%$ & $2.0 \%$ & $1.5 \%$ & $1.5 \%$ & $1.5 \%$ \\
\hline Central Africa & $2.1 \%$ & $2.6 \%$ & $2.9 \%$ & $2.8 \%$ & $2.7 \%$ & $2.0 \%$ & $1.8 \%$ & $2.2 \%$ & $1.9 \%$ & $1.8 \%$ & $2.4 \%$ & $2.6 \%$ & $2.0 \%$ & $1.5 \%$ & $1.5 \%$ & $1.1 \%$ & $1.5 \%$ \\
\hline Eastern Africa & $2.8 \%$ & $2.9 \%$ & $3.0 \%$ & $2.7 \%$ & $2.6 \%$ & $3.5 \%$ & $2.8 \%$ & $2.1 \%$ & $1.8 \%$ & $1.7 \%$ & $2.2 \%$ & $3.5 \%$ & $2.7 \%$ & $1.8 \%$ & $1.3 \%$ & $1.2 \%$ & $1.2 \%$ \\
\hline Northern Africa & $2.6 \%$ & $2.7 \%$ & $2.6 \%$ & $1.9 \%$ & $1.7 \%$ & $3.1 \%$ & $3.9 \%$ & $4.1 \%$ & $4.1 \%$ & $3.6 \%$ & $4.5 \%$ & $2.1 \%$ & $1.0 \%$ & $0.8 \%$ & $0.7 \%$ & $0.7 \%$ & $0.7 \%$ \\
\hline Southern Africa & $2.4 \%$ & $2.3 \%$ & $2.5 \%$ & $2.3 \%$ & $1.3 \%$ & $2.6 \%$ & $2.2 \%$ & $2.1 \%$ & $2.4 \%$ & $2.0 \%$ & $2.5 \%$ & $4.9 \%$ & $4.2 \%$ & $3.4 \%$ & $3.5 \%$ & $2.3 \%$ & $3.5 \%$ \\
\hline Weste & $2.3 \%$ & $2.7 \%$ & $\%$ & & $2.6^{c}$ & $2.4 \%$ & $2.5 \%$ & $2.9 \%$ & $2.4 \%$ & $2.6 \%$ & $2.8 \%$ & $2.3 \%$ & $2.4 \%$ & $2.8 \%$ & $2.1 \%$ & $2.2 \%$ & $2.0 \%$ \\
\hline America & $2.0 \%$ & $1.8 \%$ & $1.6 \%$ & $1.5 \%$ & 1.19 & $1.3 \%$ & $1.5 \%$ & $2.0 \%$ & $2.5 \%$ & $3.4 \%$ & 3.8\% & $4.7 \%$ & $4.0 \%$ & $4.2 \%$ & $4.6 \%$ & $5.5 \%$ & $5.8 \%$ \\
\hline Caribbean & $2.0 \%$ & $1.6 \%$ & 1.4 & $1.2 \%$ & 0.9 & $7.0 \%$ & $9.4 \%$ & $11.2 \%$ & $13.4 \%$ & $15.4 \%$ & $16.3 \%$ & $2.5 \%$ & $2.8 \%$ & $2.6 \%$ & $2.5 \%$ & $2.6 \%$ & $2.1 \%$ \\
\hline Central & $3.0 \%$ & $2.8 \%$ & $2.1 \%$ & $1.8 \%$ & $1.4 \%$ & $1.7 \%$ & $2.0 \%$ & $3.5 \%$ & $5.6 \%$ & $9.0 \%$ & $10.0 \%$ & $0.9 \%$ & $0.6 \%$ & $0.5 \%$ & $0.6 \%$ & $0.8 \%$ & $0.9 \%$ \\
\hline Northern America & $1.3 \%$ & $1.1 \%$ & $1.0 \%$ & $1.2 \%$ & $0.9^{c}$ & $1.0 \%$ & $1.1 \%$ & $1.1 \%$ & $1.0 \%$ & $1.1 \%$ & $1.0 \%$ & $6.8 \%$ & $6.6 \%$ & $7.9 \%$ & $9.8 \%$ & $12.7 \%$ & $13.7 \%$ \\
\hline Sou & $2.6 \%$ & $2.3 \%$ & & & 1.2 & $0.9 \%$ & $0.9 \%$ & $1.2 \%$ & $1.5 \%$ & $1.9 \%$ & $2.5 \%$ & $3.4 \%$ & $2.4 \%$ & $1.9 \%$ & $1.4 \%$ & $1.2 \%$ & $1.1 \%$ \\
\hline Asia & $2.3 \%$ & $2.1 \%$ & $1.9 \%$ & $1.5 \%$ & 1.19 & $1.8 \%$ & $1.5 \%$ & $1.5 \%$ & $1.5 \%$ & $1.6 \%$ & $1.7 \%$ & $1.9 \%$ & $1.6 \%$ & $1.3 \%$ & $1.3 \%$ & $1.2 \%$ & $1.2 \%$ \\
\hline Central Asia & $3.1 \%$ & $2.2 \%$ & $2.0^{\circ}$ & $0.9 \%$ & 1.1 & $7.3 \%$ & $8.3 \%$ & $7.9 \%$ & $10.2 \%$ & $12.0 \%$ & $10.7 \%$ & $14.9 \%$ & $16.3 \%$ & $14.5 \%$ & $13.3 \%$ & $9.4 \%$ & $7.4 \%$ \\
\hline East Asia & $2.0 \%$ & $1.8 \%$ & $1.4 \%$ & $1.0 \%$ & $0.5 \%$ & $0.8 \%$ & $0.6 \%$ & $0.6 \%$ & $0.6 \%$ & $0.7 \%$ & $0.8 \%$ & $0.4 \%$ & $0.3 \%$ & $0.3 \%$ & $0.3 \%$ & $0.4 \%$ & $0.3 \%$ \\
\hline & $2.4 \%$ & $2.4 \%$ & & & & $3.2 \%$ & $2.4 \%$ & $2.0 \%$ & $1.7 \%$ & $1.5 \%$ & $1.6 \%$ & $3.1 \%$ & $2.2 \%$ & $1.6 \%$ & $1.1 \%$ & $0.8 \%$ & $0.6 \%$ \\
\hline South & $2.6 \%$ & $2.4 \%$ & 2.2 & $1.6 \%$ & & $0.6 \%$ & $0.6 \%$ & $0.9 \%$ & $1.4 \%$ & $1.8 \%$ & $2.1 \%$ & $1.8 \%$ & $1.3 \%$ & $0.7 \%$ & $0.6 \%$ & $0.9 \%$ & $1.0 \%$ \\
\hline Western Asia & $2.7 \%$ & $2.8 \%$ & $2.7 \%$ & $2.2 \%$ & 2.3 & $3.0 \%$ & $4.2 \%$ & $5.9 \%$ & $6.0 \%$ & $6.2 \%$ & $5.5 \%$ & $5.3 \%$ & $5.7 \%$ & $6.6 \%$ & $8.7 \%$ & $8.4 \%$ & $9.4 \%$ \\
\hline Europe & $0.8 \%$ & $0.5 \%$ & $0.4 \%$ & $0.1 \%$ & $0.2 \%$ & $7.8 \%$ & $8.0 \%$ & $7.6 \%$ & $7.7 \%$ & $7.2 \%$ & $7.4 \%$ & $4.9 \%$ & $5.8 \%$ & $6.3 \%$ & $7.2 \%$ & $7.7 \%$ & $9.2 \%$ \\
\hline Eastern Europe & $0.9 \%$ & $0.7 \%$ & & $-0.2 \%$ & $-0.3 \%$ & $10.3 \%$ & $10.2 \%$ & $9.6 \%$ & $10.2 \%$ & $9.1 \%$ & $9.1 \%$ & $5.9 \%$ & $6.2 \%$ & $6.2 \%$ & $7.0 \%$ & $6.8 \%$ & $6.8 \%$ \\
\hline & $0.7 \%$ & & & & & $7.4 \%$ & $8.3 \%$ & $7.8 \%$ & $7.8 \%$ & $7.8 \%$ & $7.3 \%$ & $4.0 \%$ & $5.9 \%$ & $6.7 \%$ & $7.6 \%$ & $8.3 \%$ & $10.7 \%$ \\
\hline & $0.8 \%$ & 0.8 & & & & $7.9 \%$ & $9.3 \%$ & $8.3 \%$ & $7.6 \%$ & $7.5 \%$ & $8.5 \%$ & $0.8 \%$ & $1.3 \%$ & $1.9 \%$ & $2.9 \%$ & $4.5 \%$ & $9.9 \%$ \\
\hline Western Europe & $0.9 \%$ & $0.3 \%$ & $0.3 \%$ & $0.4 \%$ & $0.3 \%$ & $3.7 \%$ & $3.4 \%$ & $3.5 \%$ & $3.5 \%$ & $3.7 \%$ & $3.9 \%$ & $6.7 \%$ & $8.4 \%$ & $9.7 \%$ & $10.4 \%$ & $11.2 \%$ & $11.5 \%$ \\
\hline Oceania & $2.0 \%$ & $1.7 \%$ & $1.6 \%$ & $1.4 \%$ & $1.7 \%$ & $1.8 \%$ & $2.0 \%$ & $2.7 \%$ & $3.3 \%$ & $4.3 \%$ & $4.1 \%$ & $13.3 \%$ & $15.3 \%$ & $15.0 \%$ & $15.5 \%$ & $15.5 \%$ & $17.9 \%$ \\
\hline Austra & $1.9 \%$ & $1.5 \%$ & $1.4 \%$ & $1.2 \%$ & $1.5 \%$ & $2.0 \%$ & $2.1 \%$ & $2.6 \%$ & $3.2 \%$ & $4.1 \%$ & $3.9 \%$ & $15.9 \%$ & $18.4 \%$ & $18.5 \%$ & $19.6 \%$ & $20.0 \%$ & $23.8 \%$ \\
\hline & $2.4 \%$ & $2.7 \%$ & & & & $0.6 \%$ & $0.8 \%$ & $1.4 \%$ & $2.0 \%$ & $2.7 \%$ & $2.9 \%$ & $2.2 \%$ & $2.7 \%$ & $1.9 \%$ & $1.4 \%$ & $1.2 \%$ & $0.9 \%$ \\
\hline & $2.7 \%$ & $2.1 \%$ & & & & $6.9 \%$ & $4.8 \%$ & $10.8 \%$ & $8.5 \%$ & $14.7 \%$ & $12.5 \%$ & $8.0 \%$ & $6.5 \%$ & $6.2 \%$ & $12.8 \%$ & $16.8 \%$ & $16.5 \%$ \\
\hline Polynesia & $3.0 \%$ & $1.7 \%$ & $1.2 \%$ & $1.2 \%$ & $0.9 \%$ & $4.1 \%$ & $5.2 \%$ & $17.5 \%$ & $20.0 \%$ & $26.7 \%$ & $25.9 \%$ & $2.1 \%$ & $3.2 \%$ & $5.5 \%$ & $6.5 \%$ & $6.7 \%$ & $5.5 \%$ \\
\hline Worla & $2.0 \%$ & $1.9 \%$ & $1.7 \%$ & $1.4 \%$ & $1.2 \%$ & $3.0 \%$ & $2.8 \%$ & $2.7 \%$ & $2.6 \%$ & $2.7 \%$ & $2.8 \%$ & $3.0 \%$ & $2.8 \%$ & $2.7 \%$ & $2.6 \%$ & $2.7 \%$ & $2.8 \%$ \\
\hline
\end{tabular}




\subsection{Correlations between international migration and urbanisation processes}

The relationships between the processes of urbanisation and international migration were examined by conducting correlation analyses between these variables at the international level on our panel database. Table 3 displays the correlation coefficients between migration (emigration rate at origin and immigration rate at destination) and urbanisation rate variables calculated from the raw data, or from the data after removal of time and/or country effects, ${ }^{4}$ for all sized cities and separately for small/medium-sized and large cities.

Table 3. Correlation coefficients between countries' migration and urbanisation rates. 1960-2010

\begin{tabular}{|l|c|c|c|c|c|c|c|c|}
\cline { 2 - 10 } \multicolumn{1}{l|}{} & \multicolumn{3}{c|}{ Emigration rate at Origin } & \multicolumn{4}{c|}{ Immigration rate at Destination } \\
\hline & Raw data & $\begin{array}{c}\text { Removing } \\
\text { time } \\
\text { effects }\end{array}$ & $\begin{array}{c}\text { Removing } \\
\text { country } \\
\text { effects }\end{array}$ & $\begin{array}{c}\text { Removing } \\
\text { time and } \\
\text { country } \\
\text { effects }\end{array}$ & Raw data & $\begin{array}{c}\text { Removing } \\
\text { time } \\
\text { effects }\end{array}$ & $\begin{array}{c}\text { Removing } \\
\text { country } \\
\text { effects }\end{array}$ & $\begin{array}{c}\text { Reving } \\
\text { time and } \\
\text { country } \\
\text { effects }\end{array}$ \\
\hline Population Growth & $-0.1236^{*}$ & $-0.1161^{*}$ & -0.0437 & -0.0246 & $0.2325^{*}$ & $0.2536^{*}$ & $-0.0675^{*}$ & -0.0154 \\
\hline Urbanisation rate & $0.0945^{*}$ & $0.0859^{*}$ & 0.0336 & -0.0119 & $0.5024^{*}$ & $0.5095^{*}$ & $0.1384^{*}$ & 0.0383 \\
\hline $\begin{array}{l}\text { Urbanisation rate - } \\
1 \text { Million }\end{array}$ & $-0.0823^{*}$ & $-0.0865^{*}$ & 0.0265 & 0.0035 & $0.2034^{*}$ & $0.1998^{*}$ & $-0.0591^{*}$ & $-0.1406^{*}$ \\
\hline $\begin{array}{l}\text { Urbanisation rate - } \\
\text { Small and medium- } \\
\text { size cities }\end{array}$ & $0.1657^{*}$ & $0.1605^{*}$ & 0.0288 & -0.0144 & $0.4122^{*}$ & $0.4129^{*}$ & $0.1830^{*}$ & $0.1138^{*}$ \\
\hline $\begin{array}{l}\text { Urbanisation } \\
\text { Growth rate }\end{array}$ & -0.0542 & -0.0505 & -0.0286 & -0.0213 & $-0.0964^{*}$ & $-0.0874^{*}$ & $-0.0963^{*}$ & -0.0534 \\
\hline $\begin{array}{l}\text { Urbanisation rate - } \\
1 \text { Million - Growth } \\
\text { rate }\end{array}$ & -0.038 & -0.0373 & -0.0019 & -0.0002 & $-0.0817^{*}$ & $-0.0761^{*}$ & -0.0067 & 0.0186 \\
\hline $\begin{array}{l}\text { Urbanisation rate - } \\
\text { Small and medium- } \\
\text { size cities - Growth } \\
\text { rate }\end{array}$ & -0.0356 & -0.0312 & -0.0275 & -0.0205 & -0.0559 & -0.0479 & $-0.0925^{*}$ & -0.0612 \\
\hline
\end{tabular}

Note: asterisks indicate statistical significance at $5 \%$.

Population growth was positively correlated with immigration rates and negatively correlated with emigration rates. The sign and significance of this relationship persists when the time effect is removed, but disappears when country effects are not present. Consequently, the observed correlation is a country-effect issue, i.e. countries with higher population growth are also those with less emigration and more immigration. On the other hand, urbanisation rates are positively correlated with both emigration and immigration rates, and again the country effect dominates, so the more urbanised countries are the ones with a higher propensity to international migration. However, and interestingly enough, we find conflicting signs for the different urbanisation rates

\footnotetext{
${ }^{4}$ In order to remove country and time effects we regressed every variable against time and/or country fixed effects: $Y_{i t}=\varphi_{i}+\phi_{t}+\varepsilon_{i t}$. The residuals of every regression are used to compute the corrected correlations. More details of this strategy can be seen in Daveri and Tabellini (2000).
} 
with respect to emigration; it is negatively correlated with urbanisation in cities of more than one million, while it is positively correlated with urbanisation in small and medium-sized cities. Similar conflicting signs are found with respect to immigration when we remove time and country effects. In other words, apparently international immigration is being directed more to smaller cities than to larger ones. Finally, correlations between growth in urbanisation rates and migration rates are generally not significant. The only exception is a significantly negative correlation between urbanisation growth in large cities and immigration: countries with higher growth of large cities (often, developing countries) seem to be those experiencing smaller international immigration rates.

Table 4. Correlation coefficients between countries' migration and urbanisation rates, separately for developed (top table) and developing countries (bottom table). 1960-2010.

\begin{tabular}{|l|c|c|c|c|c|c|c|c|}
\cline { 2 - 10 } \multicolumn{1}{l|}{} & \multicolumn{4}{c|}{ Emigration rate at Origin } & \multicolumn{4}{c|}{ Immigration rate at Destination } \\
\hline Developed countries & Raw data & $\begin{array}{c}\text { Removing } \\
\text { time } \\
\text { effects }\end{array}$ & $\begin{array}{c}\text { Removing } \\
\text { country } \\
\text { effects }\end{array}$ & $\begin{array}{c}\text { Removing } \\
\text { time and } \\
\text { country } \\
\text { effects }\end{array}$ & Raw data & $\begin{array}{c}\text { Removing } \\
\text { time } \\
\text { effects }\end{array}$ & $\begin{array}{c}\text { Removing } \\
\text { country } \\
\text { effects }\end{array}$ & $\begin{array}{c}\text { Removing } \\
\text { time and } \\
\text { country } \\
\text { effects }\end{array}$ \\
\hline Population Growth & -0.087 & -0.0377 & $-0.1727^{*}$ & -0.0759 & $0.1867^{*}$ & $0.2758^{*}$ & 0.0031 & $0.2587^{*}$ \\
\hline Urbanisation rate & $-0.1602^{*}$ & $-0.2072^{*}$ & $0.1749^{*}$ & 0.0653 & $0.3731^{*}$ & $0.3432^{*}$ & $0.2478^{*}$ & $-0.2070^{*}$ \\
\hline $\begin{array}{l}\text { Urbanisation rate - } \\
\text { 1 Million }\end{array}$ & $-0.3273^{*}$ & $-0.3332^{*}$ & 0.0495 & -0.0177 & -0.0378 & -0.0486 & $0.1674^{*}$ & -0.0447 \\
\hline $\begin{array}{l}\text { Urbanisation rate - Small } \\
\text { and medium-size cities }\end{array}$ & 0.0932 & 0.0694 & $0.1771^{*}$ & 0.0749 & $0.3590^{*}$ & $0.3336^{*}$ & $0.2218^{*}$ & $-0.1905^{*}$ \\
\hline Urbanisation Growth rate & 0.0308 & 0.0811 & -0.0442 & 0.0446 & -0.0437 & 0.0053 & -0.0757 & 0.107 \\
\hline $\begin{array}{l}\text { Urbanisation rate - } \\
\text { 1 Million - Growth rate }\end{array}$ & -0.019 & -0.013 & 0.0733 & 0.0926 & -0.0538 & -0.0346 & -0.1265 & -0.0494 \\
\hline $\begin{array}{l}\text { Urbanisation rate - Small } \\
\text { and medium-size cities - } \\
\text { Growth rate }\end{array}$ & 0.0393 & 0.0863 & -0.071 & 0.0113 & -0.0241 & 0.0198 & -0.0375 & 0.1252 \\
\hline
\end{tabular}

\begin{tabular}{|l|c|c|c|c|c|c|c|c|}
\cline { 2 - 10 } \multicolumn{1}{c|}{} & \multicolumn{4}{c|}{ Emigration rate at Origin } & \multicolumn{4}{c|}{ Immigration rate at Destination } \\
\hline Developing countries & Raw data & $\begin{array}{c}\text { Removing } \\
\text { time } \\
\text { effects }\end{array}$ & $\begin{array}{c}\text { Removing } \\
\text { country } \\
\text { effects }\end{array}$ & $\begin{array}{c}\text { Removing } \\
\text { time and } \\
\text { country } \\
\text { effects }\end{array}$ & Raw data & $\begin{array}{c}\text { Removing } \\
\text { time } \\
\text { effects }\end{array}$ & $\begin{array}{c}\text { Removing } \\
\text { country } \\
\text { effects }\end{array}$ & $\begin{array}{c}\text { Removing } \\
\text { time and } \\
\text { country } \\
\text { effects }\end{array}$ \\
\hline Population Growth & $-0.1348^{*}$ & $-0.1285^{*}$ & -0.0393 & -0.0231 & $0.3202^{*}$ & $0.3408^{*}$ & $-0.0768^{*}$ & -0.0464 \\
\hline Urbanisation rate & $0.1148^{*}$ & $0.1079^{*}$ & 0.0281 & -0.016 & $0.5365^{*}$ & $0.5530^{*}$ & $0.1234^{*}$ & $0.0695^{*}$ \\
\hline $\begin{array}{l}\text { Urbanisation rate - } \\
1 \text { Million }\end{array}$ & $-0.0674^{*}$ & $-0.0716^{*}$ & 0.0262 & 0.0045 & $0.2433^{*}$ & $0.2408^{*}$ & $-0.0848^{*}$ & $-0.1518^{*}$ \\
\hline $\begin{array}{l}\text { Urbanisation rate - Small } \\
\text { and medium-size cities }\end{array}$ & $0.1903^{*}$ & $0.1874^{*}$ & 0.0225 & -0.0194 & $0.4336^{*}$ & $0.4423^{*}$ & $0.1783^{*}$ & $0.1545^{*}$ \\
\hline Urbanisation Growth rate & -0.0627 & -0.0607 & -0.0304 & -0.0264 & $-0.0985^{*}$ & $-0.0941^{*}$ & $-0.1025^{*}$ & $-0.0818^{*}$ \\
\hline $\begin{array}{l}\text { Urbanisation rate }- \\
\text { 1 Million - Growth rate }\end{array}$ & -0.0389 & -0.0384 & -0.0048 & -0.0036 & $-0.0784^{*}$ & $-0.0743^{*}$ & 0.0077 & 0.0263 \\
\hline $\begin{array}{l}\text { Urbanisation rate - Small } \\
\text { and medium-size cities - } \\
\text { Growth rate }\end{array}$ & -0.0425 & -0.0402 & -0.0272 & -0.0235 & -0.0573 & -0.0543 & $-0.1043^{*}$ & $-0.0927^{*}$ \\
\hline
\end{tabular}

Note: asterisks indicate statistical significance at $5 \%$.

Because the analysis of world trends suggest that there are differences across regions according to the level of economic development, we have divided the sample into developed countries and 
developing countries and have computed the correlation coefficients separately for each ${ }^{5}$ (Table 4). When we look at the raw data the basic figures are overall similar to the global ones. The picture is different when we focus on correlations once country and time effects have been removed. We found important differences between developed and developing regions. While in developed countries population growth is significantly correlated with immigration rates, in developing countries this is not the case. On the other hand, urbanisation is negatively correlated with immigration rates in developed regions but still positively correlated in developing countries. The main driver of these differences is the urbanisation rate in small and medium-sized cities, which is negatively correlated with immigration in developed regions but positively correlated in developing countries.

\section{Regression Analysis}

In this section, the correlation analysis between migration rates and urbanisation goes a step further by controlling for other relevant factors in the urbanisation process. Thus, in order to test the influence on international migration of urbanisation rates we followed the parametric strategy proposed in Barrios et al. (2006):

$$
\operatorname{Urban}_{i t}=\alpha+\beta \boldsymbol{X}_{i t}+\delta_{t}+u_{i}+v_{i t}
$$

where $U_{r b a n}$ it is the urbanisation rate, $\boldsymbol{X}_{i t}$ is a vector of time-varying potential determinants, in which we include migration rates, $\delta_{t}$ are time-specific effects common to all countries, $u_{i}$ are timeinvariant country-specific effects, and $v_{i t}$ is the usual error term. We did not aim to perform a strict causality analysis but rather to inspect the correlation between urbanisation and international migration once other factors had been cleared, taking advantage of the panel structure of the database, and performing a set of cross-country regression analyses.

For Urban, our dependent variable, we considered three different rates: urbanisation, urbanisation in larger cities ( $>1$ million inhabitants), and urbanisation in smaller cities ( $<1$ million inhabitants). For migration rates we included immigration rate at destination [immigr] and the emigration rate at origin [emigr]. As controls, we included a list of classical factors in the applied literature: two economic variables, GDP per capita $[G D P p c]$ and telephones per capita [telph_pc]; three demographic variables, total population [pop_total], the proportion of young [pop_0_14] and older people [pop_m65], and two development variables: life expectancy at birth [life_exp] and infant

\footnotetext{
${ }^{5}$ In order to classify every country as developed or developing, we have followed the United Nations composition of economic regions, available at http://unstats.un.org/unsd/methods/m49/m49regin.htm\#ftnc. Developed countries are the ones included in the following regions: Europe, North America, Japan, Australia and New Zealand.
} 
mortality rates [mort_inf]. Even though we assumed that there may be relevant factors that we had excluded from the controls considered, for instance those related to institutions, we deemed that these factors would be mostly captured by development variables or, at a later stage, in the fixed effects specification. Even though these country-specific effects represent a measure of our ignorance, they are a good control for time invariant omitted variables.

The empirical model introduces all variables in logs, except the ones expressed as percentages, and is summarised in the following equation:

$$
\begin{aligned}
& \operatorname{Urban}_{i t}=\beta_{0}+\beta_{1} \text { immigr }_{i t}+\beta_{2} \text { emigr }_{i t}+\beta_{3} \ln G D P p c_{i t}+\beta_{4} \ln t e l p h \_p c_{i t} \\
& +\beta_{5} \ln \text { pop_total } i t+\beta_{6} \text { pop_0_14 }{ }_{i t}+\beta_{7} \text { pop_m6 } 65_{i t}+\beta_{8} \ln \text { life_exp } p_{i t} \\
& +\beta_{9} \ln \text { mort_inf } f_{i t}+\delta_{t}+u_{i}+v_{i t}
\end{aligned}
$$

We calculated the between estimates (BE), which can be interpreted as measuring the long-run effects on urbanisation rates, and the fixed-effects estimates (FE), which capture how time-series changes within a country impinge on the changes in its urbanisation rate over time. In addition, given that these coefficients only reflect within-country time-series variation, they can be interpreted as short-run effects. We also performed a pooled estimation ('Pool'), which can be interpreted as an average of the $\mathrm{BE}$ and FE estimates. Table 5 displays the descriptive statistics of the main variables of our analysis. Most of the information on urbanisation rates and immigration rates at destination is cross sectional. Consequently, we expected that the BE estimates captured a substantial part of the variation of the urbanisation variables, while the FE results would explain the variations observed within countries over the 50 years considered in our study.

Table 5. Descriptive statistics of main variables in the regression analysis

\begin{tabular}{|l|c|c|c|c|}
\hline \multirow{2}{*}{} & \multirow{2}{*}{ Mean } & \multicolumn{3}{|c|}{ Standard Deviation } \\
\cline { 3 - 5 } & & overall & between & within \\
\hline Emigration rate at Origin & 9.07 & 23.67 & 12.78 & 19.94 \\
\hline Immigration rate at Destination & 7.33 & 11.53 & 10.62 & 4.55 \\
\hline Population Growth & 1.870 & 1.444 & 1.122 & 0.913 \\
\hline Urbanisation rate & 47.78 & 25.01 & 23.28 & 9.27 \\
\hline Urbanisation rate - 1 Million & 11.62 & 16.38 & 16.12 & 3.07 \\
\hline $\begin{array}{l}\text { Urbanisation rate - Small and medium-size } \\
\text { cities }\end{array}$ & 36.16 & 22.40 & 20.96 & 8.00 \\
\hline Urbanisation Growth rate & 4.361 & 4.315 & 2.789 & 3.297 \\
\hline Urbanisation rate - 1 Million - Growth rate & 0.840 & 2.209 & 1.484 & 1.639 \\
\hline $\begin{array}{l}\text { Urbanisation rate - Small and medium-size } \\
\text { cities - Growth rate }\end{array}$ & & & & \\
\hline
\end{tabular}


In order to obtain robust estimates of the standard errors, the estimates relaxed the usual requirement that the observations are independent, and consequently we allowed for intragroup correlation in the disturbance term: the observations are independent across groups (clusters) but not necessarily within groups. The considered groups are the world's subregions. The results are displayed in table 6.

Table 6. Regression results. Parameter estimates. Full database.

\begin{tabular}{|c|c|c|c|c|c|c|c|c|c|}
\hline & \multicolumn{3}{|c|}{ Urbanisation rate } & \multicolumn{3}{|c|}{ Urbanisation rate - 1 Million } & \multicolumn{3}{|c|}{$\begin{array}{c}\text { Urbanisation rate - Small and } \\
\text { medium-size cities }\end{array}$} \\
\hline & $\begin{array}{c}\text { Pooled } \\
\text { OLS }\end{array}$ & Between & $\begin{array}{l}\text { Fixed } \\
\text { Effects }\end{array}$ & $\begin{array}{c}\text { Pooled } \\
\text { OLS }\end{array}$ & Between & $\begin{array}{c}\text { Fixed } \\
\text { Effects }\end{array}$ & $\begin{array}{l}\text { Pooled } \\
\text { OLS }\end{array}$ & Between & $\begin{array}{c}\text { Fixed } \\
\text { Effects }\end{array}$ \\
\hline Immig & $0.354 * * *$ & $0.373 * * *$ & $0.299 * * *$ & 0.288 & $0.268 * *$ & -0.152 & $0.279 * * *$ & 0.105 & $0.314 * * *$ \\
\hline Emig & -0.106 & -0.240 & -0.020 & 0.111 & 0.020 & 0.011 & -0.119 & -0.260 & -0.031 \\
\hline $\ln$ GDP pc & $3.871 * * *$ & $7.530 * * *$ & 1.894 & 0.66 & $3.936 * *$ & 0.015 & 2.232 & $3.594 *$ & 1.879 \\
\hline ln Telph_pc & $2.702 * * *$ & $6.126 * * *$ & $2.219 * *$ & 0.19 & 2.896 & 0.203 & $2.500 * * *$ & 3.229 & $2.016 * *$ \\
\hline Pop_total & $2.844 * * *$ & 1.164 & $12.531 * * *$ & $3.586 * * *$ & $4.737 * * *$ & $4.202 * *$ & -0.432 & $-3.573 * * *$ & $8.329 * * *$ \\
\hline pop_0_14 & -0.17 & -0.299 & -0.096 & -0.007 & 0.578 & -0.017 & $-0.175^{*}$ & $-0.877 * *$ & -0.079 \\
\hline pop_m65 & -0.132 & 0.143 & 0.307 & $0.242 * *$ & -0.287 & $0.358 * *$ & -0.319 & 0.43 & -0.05 \\
\hline ln life_exp & $20.571 * * *$ & 1.949 & 6.91 & $5.124^{*}$ & 3.348 & 3.899 & $13.827 * *$ & -1.399 & 3.01 \\
\hline ln mort_inf & 2.792 & $7.714 * *$ & 1.489 & -0.394 & 0.29 & -0.447 & $3.030 * *$ & $7.424 *$ & 1.936 \\
\hline 1970 & 1.905 & & 0.807 & 0.478 & & 0.538 & $1.687^{*}$ & & 0.269 \\
\hline 1980 & $2.920 *$ & & 1.083 & 0.121 & & 0.203 & $3.267 * * *$ & & 0.88 \\
\hline 1990 & $4.997 * *$ & & 1.748 & -0.311 & & -0.29 & $5.828 * * *$ & & 2.037 \\
\hline 2000 & $5.411 *$ & & 0.952 & -0.996 & & -1.127 & $6.893 * * *$ & & 2.078 \\
\hline 2010 & $6.213 * *$ & & 1.144 & -1.645 & & -1.759 & $8.520 * * *$ & & 2.903 \\
\hline Constant & $-121.9 * * *$ & -59.58 & $-200.1 * * *$ & $-69.06 * * *$ & -129.18 & $-68.41 * *$ & $-43.186 *$ & 69.592 & $-131.74 * * *$ \\
\hline $\mathrm{N}$ & 739 & 739 & 739 & 739 & 739 & 739 & 739 & 739 & 739 \\
\hline $\mathrm{R}^{2}$ & 0.650 & 0.701 & 0.744 & 0.251 & 0.396 & 0.352 & 0.443 & 0.505 & 0.664 \\
\hline $\operatorname{Adj} R^{2}$ & 0.639 & 0.684 & 0.739 & 0.234 & 0.362 & 0.339 & 0.425 & 0.477 & 0.657 \\
\hline
\end{tabular}

Note: asterisks indicate statistical significance at $1 \%(* * *), 5 \%(* *)$ and $10 \%(*)$.

Immigration rates displayed a positive and significant parameter in all estimates (pooled, between and fixed effects) for the global urbanisation rate, in the between-estimate for larger cities, and in the pooled and fixed effects estimates for the small and medium-sized cities (Table 5). Thus, immigration is clearly linked to within-country increases in urbanisation rates, and this is particularly true for small and medium-sized cities. Quantitatively, the fixed effects estimates report that a $1 \%$ increase in the immigration rate is associated to a $0.3 \%$ higher urbanisation rate in the small and medium-sized cities

The regression of urbanisation on emigration had a negative, although non-significant sign, as was found for bivariate correlation analysis (see section 3.2). In other words: countries with higher (between estimates) or increasing (fixed effects estimates) emigration rates did not experience smaller or decreasing levels of urbanisation. 
The control variables included in the model provided the following results:

1. A global trend in urbanisation was identified, captured by the individual time dummies, which was independent of other factors and statistically significant in small and medium-sized cities. The trend was non-significant for larger cities.

2. Social and economic development is relevant for explaining differences in urbanisation rates between countries, but not over time (the fixed effect estimates were never significant). Only telephones per capita displayed a significant result in small and medium-sized cities, suggesting an important role of connectivity for smaller cities.

3. Population size was significant for almost all estimates. The positive parameter in the fixed effects specification indicates that countries with faster population growth are the ones that experience faster increases in urbanisation rates. We found that on average (between estimates), more populated countries had a smaller proportion of small and medium-sized cities, possibly associated with the trend of growing megacities in many large developing countries such as Brazil, India and China.

4. Even though the demographic structure seems to play a minor role, we did find a number of significant parameters. On the one hand, on average (between estimates) countries with higher urbanisation rates in small and medium-sized cities displayed a negative relationship with the proportion of younger people. On the other hand, countries with a higher proportion of older people saw increases (fixed effects estimates) in the urbanisation rate in larger cities.

To explore in more depth these results, we divided the full sample of countries into economically developed and developing countries. We have seen that developed countries receive more immigrants, while developing countries have more emigrants, leading to expect a different structure of the model for these two groups of countries. On the other hand, as we have also seen there is a certain divide in population trends over the period considered, i.e. in recent periods urbanisation has accelerated, while migration rates have also increased. As most estimates show similar results for the control variables, table 7 only displays the parameters related with international migration.

From this analysis on the developed-developing divide, results show that larger international immigration rates are linked to higher urbanisation rates, particularly for small and medium-sized cities, but also for larger cities (between estimate in developing countries). Contrary to what was found when looking at bivariate correlations, in the regression analysis, once all controls have been taken into account, immigration was not significantly negative associated with urbanisation in larger cities. 
Emigration was significant and negative in developing regions, but not so in the developed regions. This result suggests, at least from several estimates, that international emigration from developing countries occurs at the expense of local urbanisation: an emigration rate $1 \%$ higher is associated to a $0.16 \%$ (pool estimate for small and medium-sized cities) to $0.34 \%$ (between estimates for the global urbanisation rate) lower urbanisation rate. Our interpretation of this result is that migrants consider as destination both national as well as international urban destinations.

The 1960-1980 period displayed substantially less significant results than the 1990-2010 period. The impact of international migration on urbanisation has been more pronounced in recent decades (i.e. 1990-2010) than earlier (1960-1980). And this is true for both immigration and emigration. We see that the quantitative impact of immigration on urbanisation rates in smaller cities has decreased over time (from $0.31 \%$ in the $1960-1980$ period to $0.17 \%$ in the $1990-2010$ period), probably as a consequence of a large increase in urbanisation rates in the smaller cities in developing countries.

Table 7. Regression results. Parameter estimates. Subsamples according to development and time

\begin{tabular}{|c|c|c|c|c|c|c|c|c|}
\hline \multicolumn{3}{|c|}{ Urbanisation rate } & \multicolumn{3}{|c|}{$\begin{array}{c}\text { Urbanisation rate - } 1 \\
\text { Million } \\
\end{array}$} & \multicolumn{3}{|c|}{$\begin{array}{c}\text { Urbanisation rate - Small and } \\
\text { medium-size cities }\end{array}$} \\
\hline $\begin{array}{l}\text { Pooled } \\
\text { OLS }\end{array}$ & Between & $\begin{array}{l}\text { Fixed } \\
\text { Effects }\end{array}$ & $\begin{array}{l}\text { Pooled } \\
\text { OLS }\end{array}$ & Between & $\begin{array}{c}\text { Fixed } \\
\text { Effects }\end{array}$ & $\begin{array}{l}\text { Pooled } \\
\text { OLS }\end{array}$ & Between & $\begin{array}{l}\text { Fixed } \\
\text { Effects }\end{array}$ \\
\hline
\end{tabular}

Developed

\begin{tabular}{|l|ccc|ccc|ccc|}
\hline Immgr. rate & $0.372 * * *$ & 0.066 & $0.302 * * *$ & -0.018 & 0.199 & -0.064 & $0.378 * * *$ & -0.133 & $0.365 * * *$ \\
Emigr. rate & 0.096 & -0.394 & 0.134 & 0.027 & 0.138 & 0.030 & 0.083 & -0.532 & 0.103 \\
\hline
\end{tabular}

Developing

\begin{tabular}{|l|ccc|ccc|ccc|}
\hline Immgr. rate & $0.402 * * *$ & $0.432 * * *$ & $0.345 * * *$ & 0.066 & $0.392 * * *$ & -0.001 & $0.279 * * *$ & 0.039 & $0.346 * * *$ \\
Emigr. rate & -0.163 & $-0.341 *$ & -0.086 & -0.007 & -0.258 & -0.029 & $-0.159 *$ & -0.083 & -0.057 \\
\hline
\end{tabular}

All countries. 1960-1980

\begin{tabular}{|l|ccc|ccc|ccc|}
\hline Immgr. rate & $0.404 * *$ & 0.218 & 0.301 & 0.094 & 0.231 & -0.001 & $0.279 * *$ & -0.013 & $0.307 *$ \\
Emigr. rate & 0.004 & -0.073 & 0.149 & 0.019 & 0.075 & 0.011 & -0.001 & -0.148 & 0.138 \\
\hline
\end{tabular}

All countries. 1990-2010

\begin{tabular}{|l|ccc|ccc|ccc|}
\hline Immgr. rate & $0.248 * * *$ & $0.392 * * *$ & $0.157 * * *$ & 0.028 & $0.242 * *$ & -0.009 & $0.169 * *$ & 0.150 & $0.167 * * *$ \\
Emigr. rate & -0.042 & $-0.277 *$ & 0.022 & 0.031 & 0.006 & 0.010 & -0.068 & $-0.283 *$ & 0.012 \\
\hline
\end{tabular}

Note: asterisks indicate statistical significance at $1 \%(* * *), 5 \%(* *)$ and $10 \%(*)$.

\section{Discussion and conclusions}

Individual case studies and regional comparisons analysing the impact of international migration on local cities have been common in the literature. Cross-national research, on the other hand, allows for testing general trends, as well as differences across world regions. The large database in this 
paper ( $c a 200$ countries over a 50-year period) allowed us to capture the global stylised facts of the relationships between international migrations and urbanisation, which have seldom been considered together (WDR, 2009).

We have identified strong and robust empirical relationships. Using panel estimations we have pointed out that immigration is indeed associated with increasing urbanisation, while emigration is only negatively associated with urbanisation in developing countries. Additionally, small and medium-sized cities are apparently more influenced by international migrations than larger cities, and this process has been particularly important in recent decades. This result is in line with recent calls (OECD, 2009) stressing that in many countries it can be the case that medium-sized and small agglomerations enjoy stronger opportunities for economic development ${ }^{6}$.

Our results clearly complement the traditional urbanisation studies where the structural change takes place by internal rural-urban migration processes. While there is no doubt that increasing urbanisation worldwide has become a major challenge for sustainable development, especially in developing countries but also in developed ones, the role of increasing international migrations in shaping the economic geography of countries deserves further research.

\section{References}

Ades, A., Glaeser, E. (1995). “Trade and circuses: Explaining urban giants,” Quarterly Journal of Economics, $110,195-228$.

Franch Auladell, X., Marti-Henneberg, J. and Puig-Farré, J. (2013) "Un análisis espacial de las pautas de crecimiento y concentración de la población a partir de series homogéneas: España (1877-2001)", Investigaciones Regionales, 25, 43-65.

Barca, F., McCann, P., \& Rodríguez-Pose, A. (2012). "The case for regional development intervention: Placebased versus place-neutral approaches," Journal of Regional Science 52(1), 134-152.

Barrios, S., Bertinelli, L. and Strobl, E. (2006). "Climatic change and rural-urban migration: The case of subSaharan Africa," Journal of Urban Economics, 60, 357-371

Brueckner, J. (1990). "Analyzing third world urbanization: A model with empirical evidence," Economic Development and Cultural Change, 38, 587-610.

Castells-Quintana, D. and Royuela, V. (2011). Agglomeration, Inequality and Economic Growth. IREA-WP series, no. 2011/14

Daveri, F. and Tabellini, G. (2000). "Unemployment, growth and taxation in industrial countries," Economic Policy, CEPR \& CES \& MSH, 15(30), 47-104, 04.

\footnotetext{
${ }^{6}$ In any case re recognise that these dynamics are not simple. In a recent study Martí and Rodenas (2012) found that up to $25 \%$ of foreign immigrants in Spain reallocate within the country two years after their arrival, and that the reallocation is more likely to happen if the first destination was a small municipality.
} 
Davis, J., Henderson, J.V. (2003). "Evidence on the political economy of the urbanization process," Journal of Urban Economics 53 98-125.

Firebaugh, G. (1979). "Structural determinants of urbanization in Asia and Latin America, 1950-1970," American Sociological Review, 44, 199-215.

Gollin, D., Parente, S.L., and Rofgerson, R. (2002). "The role of agriculture in development," American Economic Review, 92, 160-164.

Harris, J. R., and Todaro, M. P. (1970). "Migration, unemployment and development: a two-sector analysis," American Economic Review, 60, 126-142.

Henderson, J.V. and Wang, H.G. (2005). “Aspects of the rural-urban transformation of countries,” Journal of Economic Geography, 5, 23-42.

Henderson, J.V. and Wang, H.G. (2007). "Urbanization and city growth: The role of institutions," Regional science and urban economics, 37(3), 283-313.

Kasarda, J.D. Crenshaw, E.M. (1991). "Third World Urbanization: Dimensions, Theories, and Determinants," Annual Review of Sociology, 17, 467-501

Lewis, W. A. (1954). "Economic Development with Unlimited Supplies of Labour," Manchester School of Economic and Social Studies, 22, 139-191.

Martí Sempere, M. and Ródenas Calatayud, C. (2012) "Reemigrar en España: una aproximación a sus determinantes”, Investigaciones Regionales, 22, 105-128.

OECD (2009a) How Regions Grow, Paris. Organisation for Economic Cooperation and Development.

OECD (2009b) Regions Matter: Economic Recovery, Innovation and Sustainable Development. Paris. Organisation for Economic Cooperation and Development.

OECD (2009c) Regions at a Glance. Paris. Organisation for Economic Cooperation and Development.

Ortega, F., and G. Peri (2009). The Causes and Effects of International Migrations: Evidence from OECD Countries 1980-2005, NBER Working Paper No. 14883, National Bureau of Economic Research.

Ramos, R. (2013). Analysing migration flows from and to ENC through the MIG-SEARCH databases, WP3/01 SEARCH Working Paper. Available at http://www.ub.edu/searchproject/wpcontent/uploads/2013/01/WP 3 1.pdf.

Ranis, G. and Fei, J.C.H. (1961). "A Theory of Economic Development," American Economic Review, 51, 533-565.

Rauch, J. E. (1993). "Economic Development, Urban underemployment, and Income Inequality," Canadian Journal of Economics, 26, 901-18.

Todaro, M.P. (1976). Internal Migration in Developing Countries. Geneve. International Labour Organization.

World Bank. (2009). World Development Report 2009: Reshaping economic geography. Washington D.C: World Bank. 
Annex 1. Considered countries, classified by continents and geographical regions

(The countries classification by geographical regions corresponds to the United Nations Geoscheme, which can be accessed at http://unstats.un.org/unsd/methods/m49/m49.htm)

\begin{tabular}{ccc} 
Africa & & \\
\hline Central Africa & Eastern Africa & Southern Africa \\
Angola & Burundi & Botswana \\
Cameroon & Comoros & Lesotho \\
Central African Republic & Djibouti & Namibia \\
Chad & Eritrea & South Africa \\
Congo & Ethiopia & Swaziland \\
Equatorial Guinea & Kenya & Western Africa \\
Gabon & Madagascar & Benin \\
Sao Tome and Principe & Malawi & Burkina Faso \\
Northern Africa & Mauritius & Cape Verde \\
Algeria & Mozambique & Cote d'Ivoire \\
Egypt & Rwanda & Gambia \\
Libya & Seychelles & Ghana \\
Morocco & Somalia & Guinea \\
Sudan & Tanzania & Guinea-Bissau \\
Tunisia & Uganda & Liberia \\
& Zambia & Mali \\
& Zimbabwe & Mauritania \\
& & Niger \\
& & Nigeria \\
& & Senegal \\
& & Sierra Leone \\
& & Togo
\end{tabular}

\begin{tabular}{l} 
America \\
\hline Caribbean \\
Antigua and Barbuda \\
Aruba \\
Bahamas \\
Barbados \\
Cayman Islands \\
Cuba \\
Dominica \\
Dominican Republic \\
Grenada \\
Haiti \\
Jamaica \\
Puerto Rico \\
St Kitts and Nevis \\
St Lucia \\
St Vincent and the Grenadines \\
Trinidad and Tobago \\
Turks and Caicos Islands
\end{tabular}

$\begin{array}{cl}\text { Central America } & \text { South America } \\ \text { Belize } & \text { Argentina } \\ \text { Costa Rica } & \text { Bolivia } \\ \text { El Salvador } & \text { Brazil } \\ \text { Guatemala } & \text { Chile } \\ \text { Honduras } & \text { Colombia } \\ \text { Mexico } & \text { Ecuador } \\ \text { Nicaragua } & \text { Guyana } \\ \text { Panama } & \text { Paraguay } \\ \text { Northern America } & \text { Peru } \\ \text { Bermuda } & \text { Suriname } \\ \text { Canada } & \text { Uruguay } \\ \text { Greenland } & \text { Venezuela } \\ \text { United States } & \end{array}$




\begin{tabular}{|c|c|c|}
\hline \multicolumn{3}{|l|}{ Asia } \\
\hline Central Asia & East Asia & Western Asia \\
\hline Kazakhstan & China & Armenia \\
\hline Kyrgyzstan & Hong Kong & Azerbaijan \\
\hline Tajikistan & Japan & Bahrain \\
\hline Turkmenistan & Korea, North & Cyprus \\
\hline Uzbekistan & Korea, South & Georgia \\
\hline South Asia & Macao & Iraq \\
\hline Afghanistan & Mongolia & Israel \\
\hline Bangladesh & Southeast Asia & Jordan \\
\hline Bhutan & Brunei & Kuwait \\
\hline India & Cambodia & Lebanon \\
\hline Iran & Indonesia & Oman \\
\hline Maldives & Laos & Qatar \\
\hline Nepal & Malaysia & Saudi Arabia \\
\hline Pakistan & Myanmar & Syria \\
\hline Sri Lanka & Philippines & Turkey \\
\hline & Singapore & United Arab Emirates \\
\hline & Thailand & Yemen, North \\
\hline & Vietnam & \\
\hline \multicolumn{3}{|l|}{ Europe } \\
\hline Eastern Europe & Northern Europe & Southern Europe \\
\hline Belarus & Denmark & Albania \\
\hline Bulgaria & Estonia & Bosnia and Herzegovina \\
\hline Czech Republic & Faroe Islands & Croatia \\
\hline Hungary & Finland & Gibraltar \\
\hline Moldova & Iceland & Greece \\
\hline Poland & Ireland & Italy \\
\hline Romania & Latvia & Macedonia \\
\hline Russia & Lithuania & Malta \\
\hline Slovakia & Norway & Portugal \\
\hline Ukraine & Sweden & San Marino \\
\hline Western Europe & United Kingdom & Slovenia \\
\hline Austria & & Spain \\
\hline Belgium & & \\
\hline France & & \\
\hline Germany & & \\
\hline Luxembourg & & \\
\hline Netherlands & & \\
\hline Switzerland & & \\
\hline
\end{tabular}

\section{Oceania}

$\begin{array}{lll}\text { Australia and New Zealand } & \text { Micronesia } & \text { Polynesia } \\ \text { Australia } & \text { Kiribati } & \text { French Polynesia } \\ \text { New Zealand } & \text { Marshall Islands } & \text { Samoa } \\ \text { Melanesia } & \text { Micronesia } & \text { Tonga } \\ \text { Fiji } & \text { Northern Mariana Islands } & \text { Tuvalu } \\ \text { New Caledonia } & \text { Palau } & \\ \text { Papua New Guinea } & & \\ \text { Solomon Islands } & & \\ \text { Vanuatu } & & \end{array}$

